\title{
Erratum to: Role of trade-off between sexual and vertical routes for evolution of pathogen transmission
}

\author{
Veronika Bernhauerová $^{1} \cdot$ Luděk Berec $^{2,3}$
}

Published online: 28 July 2016

(C) Springer Science+Business Media Dordrecht 2016

Erratum to: Theor Ecol (2015) 8:23-36

DOI 10.1007/s12080-014-0234-8

The electronic supplementary materials (Online Resource 1 and Online Resource 2) of this article were incorrect. The updated Online Resources are now found in the link given below.

The online version of the original article can be found at http://dx.doi. org/10.1007/s12080-014-0234-8.

Electronic supplementary material The online version of this article (doi:10.1007/s12080-016-0308-x) contains supplementary material, which is available to authorized users.

Veronika Bernhauerová

bernhauerv@gmail.com

1 Department of Mathematics and Statistics, Masaryk University, Kotlářská 2, 61137 Brno, Czech Republic

2 Department of Theoretical Ecology, Institute of Entomology, Biology Centre ACR, Branišovská 31, 37005 České Budějovice, Czech Republic

3 Institute of Mathematics and Biomathematics, Faculty of Science, University of South Bohemia, Branišovská 31, 37005 České Budějovice, Czech Republic 